\title{
V392 ORIONIS: OBSERVATIONS AND EVOLUTIONARY STATE OF A LOW- MASS SYSTEM
}

\author{
S. NARUSAWA \\ Nishi-Harima Astronomical Observatory, Japan \\ narusawa@nhao.go.jp \\ A. YAMASAKI \\ National Defence Academy, Japan \\ yamasaki@cc.nda.ac.jp \\ AND \\ Y.NAKAMURA \\ Fukushima University, Japannakamura@cosmos.educ.fukushima-u.ac.jp
}

\begin{abstract}
Although the evolution of binary systems has been qualitatively interpreted with the evolutionary scenario, the quantitative interpretation of any observed system is still unsatisfactory due to the difficulty of the quantitative treatment of mass and angular momentum transfer/loss. To reach a true understanding of the evolution of binary systems, we have to accumulate more observational evidence. So far, we have observed several binaries that are short-period and noncontact, and found the existence of extremely small-mass systems. In the present paper, we study another short-period $(\mathrm{P}=0.659 \mathrm{~d})$, noncontact, eclipsing binary system, V392 Ori. We have made photometric and spectroscopic observations of V392 Ori. The light curves are found to vary, suggesting the existence of circumstellar matter around the system. Combining the photometric and spectroscopic results, we obtain parameters describing the system; we find the mass of the primary component is only $0.6 M_{\odot}$ - undermassive for its spectral and luminosity class A5V, suggesting that a considerable amount of its original mass has been lost from the system during the course of evolution. The low-mass problem is very important for investigation of the evolution of close binary systems: large mass loss within and/or after the main-sequence will have a significant influence on the future evolution of binary systems.
\end{abstract}

